$\int \ddot{O} \mathrm{~B}$

Jahrbuch der Österreichischen Byzantinistik 
Hauptherausgeber / Editor-in-chief

\section{EWALD KISLINGER}

Institut für Byzantinistik und Neogräzistik der Universität Wien

A-1010 Wien, Postgasse 7-9, 3. Stock

Ewald.Kislinger@univie.ac.at

\section{Herausgebergremium / Associate Editors}

\section{Christian Gastgeber}

JOHANNES KoDER

ANDREAS RHOBY

Institut für Mittelalterforschung, Abteilung Byzanzforschung der Österreichischen Akademie der Wissenschaften

A-1040 Wien, Wohllebengasse 12-14, 3. Stock

Christian.Gastgeber@oeaw.ac.at

Johannes.Koder@oeaw.ac.at

Andreas.Rhoby@oeaw.ac.at

\section{Administrative Assistenz / Administrative Assistants}

\section{Petra Greger}

Institut für Byzantinistik und Neogräzistik der Universität Wien

A-1010 Wien, Postgasse 7-9, 3. Stock

Petra.Greger@univie.ac.at

\section{INGRID WEICHSELBAUM}

Institut für Mittelalterforschung, Abteilung für Byzanzforschung der Österreichischen Akademie der Wissenschaften A-1040 Wien, Wohllebengasse 12-14, 3. Stock

Ingrid.Weichselbaum@oeaw.ac.at

\section{Wissenschaftlicher Beirat / Advisory Board}

Catherine Abadie-Reynal (Nancy), Wolfram Brandes (Frankfurt/M.),

Salvatore Cosentino (Bologna), Arne Effenberger (Berlin), John Haldon (Princeton),

Wolfram Hörandner (Wien), Elizabeth Jeffreys (Oxford), Taxiarches Kolias (Athen),

Sofia Kotzabassi (Thessalonike), Otto Kresten (Wien), Ralf-Johannes Lilie (Berlin),

Günter Prinzing (Mainz), Alice-Mary Talbot (Dumbarton Oaks),

Maciej Salamon (Krakau), Werner Seibt (Wien)

\section{Internet-Homepage}

www.oeaw.ac.at/byzanz/joeb

www.byzneo.univie.ac.at/

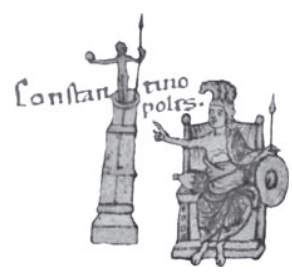




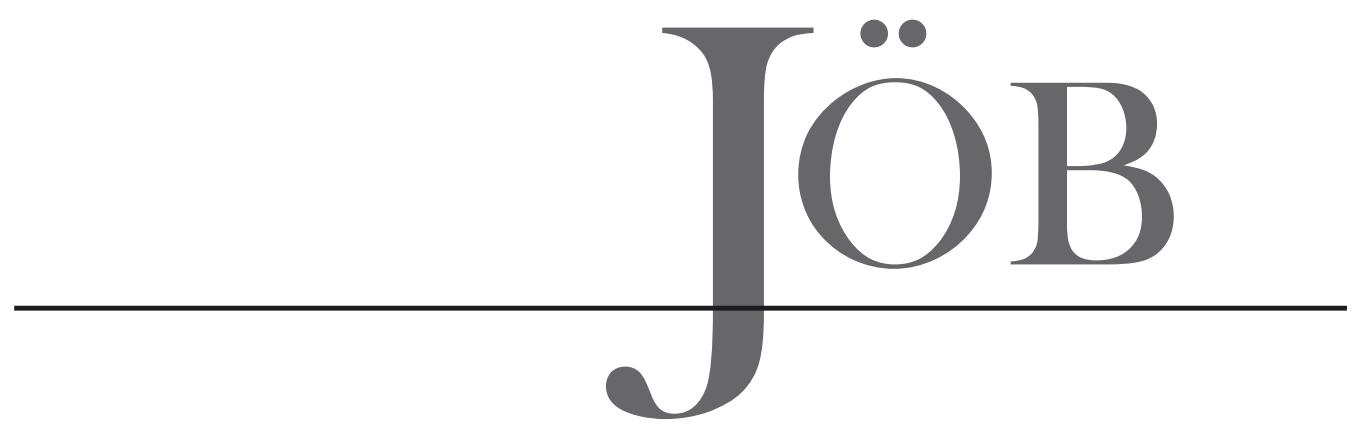

Jahrbuch der Österreichischen Byzantinistik

Band 62 / 2012

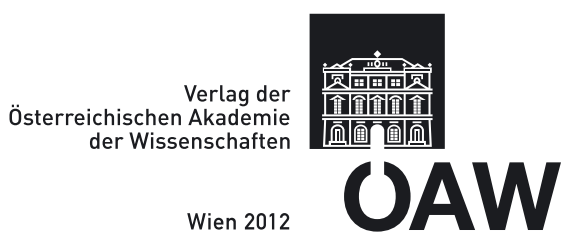




\section{Jahrbuch der Österreichischen Byzantinistik}

Herausgegeben vom Institut für Mittelalterforschung, Abteilung Byzanzforschung der Österreichischen Akademie der Wissenschaften und dem Institut für Byzantinistik und Neogräzistik der Universität Wien

Fortsetzung des Jahrbuches der Österreichischen Byzantinischen Gesellschaft

Gedruckt mit Unterstützung der Historisch-Kulturwissenschaftlichen Fakultät der Universität Wien

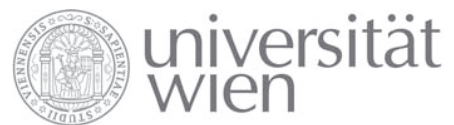

Historisch-Kulturwissenschaftliche Fakultät

Die verwendete Papiersorte ist aus chlorfrei gebleichtem Zellstoff hergestellt, frei von säurebildenden Bestandteilen und alterungsbeständig.

Alle Rechte vorbehalten

AU ISSN 0378-8660

ISBN 978-3-7001-7386-1

Copyright $@ 2012$ by

Österreichische Akademie der Wissenschaften

Wien

Satz: Maria Scherrer, Schreibbüro, Wien

Druck und Bindung: Prime Rate kft., Budapest

http://hw.oeaw.ac.at/joeb

http://verlag.oeaw.ac.at 